\title{
Biomechanical feasibility of semi-rigid stabilization and semi-rigid lumbar interbody fusion: a finite element study
}

\author{
Chia-En Wong ${ }^{1 \dagger}$, Hsuan-Teh Hu ${ }^{2,3 \dagger}$, Li-Hsing Kao ${ }^{2}$, Che-Jung Liu ${ }^{2}$, Ke-Chuan Chen ${ }^{2}$ and Kuo-Yuan Huang ${ }^{*^{*}}$ (D)
}

\begin{abstract}
Background: Semi-rigid lumbar fusion offers a compromise between pedicle screw-based rigid fixation and noninstrumented lumbar fusion. However, the use of semi-rigid interspinous stabilization (SIS) with interspinous spacer and ligamentoplasty and semi-rigid posterior instrumentation (SPI) to assist interbody cage as fusion constructs remained controversial. The purpose of this study is to investigate the biomechanical properties of semi-rigidly stabilized lumbar fusion using SIS or SPI and their effect on adjacent levels using finite element (FE) method.

Method: Eight FE models were constructed to simulate the lumbosacral spine. In the non-fusion constructs, semirigid stabilization with (i) semi-rigid interspinous spacer and artificial ligaments (PD-SIS), and (ii) PI with semi-rigid rods were simulated (PD + SPI). For fusion constructs, the spinal models were implanted with (iii) PEEK cage only (Cage), (iv) PEEK cage and SIS (Cage+SIS), (v) PEEK cage and SPI (Cage+SPI), (vi) PEEK cage and rigid PI (Cage+PI).

Result: The comparison of flexion-extension range of motion (ROM) in the operated level showed the difference between Cage+SIS, Cage+SPI, and Cage+PI was less than 0.05 degree. In axial rotation, ROM of Cage+SIS were greater than Cage+PI by 0.81 degree. In the infrajacent level, while Cage+PI increased the ROM by 24.1, 27,7, 25.9, and $10.3 \%$ and Cage+SPI increased the ROM by 26.1, 30.0, 27.1, and 10.8\% in flexion, extension, lateral bending and axial rotation respectively, Cage+SIS only increased the ROM by 3.6, 2.8, and 11.2\% in flexion, extension, and lateral bending and reduced the ROM by $1.5 \%$ in axial rotation. The comparison of the von Mises stress showed that SIS reduced the adjacent IVD stress by $9.0 \%$. The simulation of the strain energy showed a difference between constructs less than 7.9\%, but all constructs increased the strain energy in the infradjacent level.
\end{abstract}

Conclusion: FE simulation showed semi-rigid fusion constructs including Cage+SIS and Cage+SPI can provide sufficient stabilization and flexion-extension ROM reduction at the fusion level. In addition, SIS-assisted fusion resulted in less hypermobility and less von Mises stress in the adjacent levels. However, SIS-assisted fusion had a disadvantage of less $\mathrm{ROM}$ reduction in lateral bending and axial rotation. Further clinical studies are warranted to investigate the clinical efficacy and safety of semi-rigid fusions.

Keywords: Posterior lumbar interbody fusion (PLIF), Semi rigid stabilization, Semi-rigid interspinous spacer, Adjacent degeneration, Finite element

\footnotetext{
*Correspondence: hkyuan@mail.ncku.edu.tw

${ }^{\dagger}$ Chia-En Wong and Hsuan-Teh Hu contributed equally to this work.

${ }^{4}$ Department of Orthopedics, National Cheng Kung University Hospital, College of Medicine, National Cheng Kung University, Tainan, Taiwan

Full list of author information is available at the end of the article
}

\section{Background}

Posterior lumbar interbody fusion (PLIF) has been the gold standard procedure for patients with lumbar disc degeneration unresponsive to conservative treatments with excellent clinical outcomes [1-3]. In addition to the placement of interbody implants, rigid fixation using original author(s) and the source, provide a link to the Creative Commons licence, and indicate if changes were made. The images or other third party material in this article are included in the article's Creative Commons licence, unless indicated otherwise in a credit line to the material. If material is not included in the article's Creative Commons licence and your intended use is not permitted by statutory regulation or exceeds the permitted use, you will need to obtain permission directly from the copyright holder. To view a copy of this licence, visit http://creativecommons.org/licenses/by/4.0/. The Creative Commons Public Domain Dedication waiver (http://creativeco mmons.org/publicdomain/zero/1.0/) applies to the data made available in this article, unless otherwise stated in a credit line to the data. 
posterior instrumentation (PI) with pedicle screw-based construct was commonly performed in combination with PLIF to provide immediate stabilization of the spine and promote bone fusion $[4,5]$. However, rigid fixation markedly alters the biomechanics of the spine, often causing decreased motion at the operated level and a subsequent compensatory increase of motion and intradiscal pressure at the adjacent levels, which further contribute to accelerated adjacent segment degeneration (ASD) [6-8]. As a result, non-instrumented lumbar fusion has always been a well-established alternative method to instrumented PLIF $[9,10]$. In single-level lumbar disease, previous studies have shown that non-instrumented lumbar fusion provided comparable results in clinical satisfaction $[5,11]$.

More versatile implants were developed to assist and promote lumbar fusion [12-16]. Semi-rigid fusion strategies were proposed as a compromise between pedicle screw based rigid fixation and non-instrumented lumbar fusion $[17,18]$. By allowing a small-ranged, limited spinal motion, the use of semi-rigid stabilization strategies has been advocated to achieve a more physiologic bone fusion and reduce the undesirable effects following rigid fixation, such as stress shielding of the interbody space, screw loosening at the bone interface, construct fatigue, fractures of the pedicle, and ASD $[19,20]$. Semi-rigid stabilization implants included semi-rigid PI (SPI) and semirigid interspinous spacer (SIS) with ligamentoplasty [8]. Previous studies showed SPI provided comparable stability to rigid fixation while maintaining limited motions $[21,22]$. Although, the use of SPI with lumbar interbody cage were evaluated, the impact of the constructs on the superior and inferior adjacent levels remained uninvestigated $[21,23]$.

Originally designed as an implant for non-fusion lumbar surgery, the off-label use of SIS with ligamentoplasty in combination with interbody cage as a fusion construct was discussed but remained controversial [24]. Biomechanical studies showed that the combined use of SIS and ligamentoplasty could provide effective limitation in spinal motion[25-27]. The results by Tsai et al. revealed the SIS device Coflex allows limited motion that is significantly less than the motion found in the destabilized spine, and the allowed motion is not significantly different from the intact specimens[27]. The in vitro and FE study by Lafage et al. indicated a decrease of disc stresses and increase of loads transmitted to the spinous processes following SIS[28]. Gonzalez-Blohm et al. evaluated the biomechanics of the ASPEN SIS device in PLIF constructs and they suggested that the SIS device and PI performed equivalently in flexion-extension, but the pedicle screws construct was more resistant to lateral bending[29]. Moreover, compared to pedicle screw-based constructs, SIS and ligamentoplasty have several advantages in terms of smaller incision, shorter operative time, less intraoperative blood loss, and less muscle dissection [30]. The preservation of the paravertebral musculature integrity results in better soft tissue support and benefits patients' post-operative recovery [31].

Despite the potential advantages of semi-rigid implants, given the paucity of clinical evidence and lack of biomechanical evaluations, the use of semi-rigid fusion was not well-established. Although previous efforts were made to investigate lumbar fusion with SPI, critical parameters relevant to the development of ASD such as the stress and strain energy on both superior and inferior adjacent discs remained poorly-evaluated [21, 23]. Moreover, the effect of these constructs on adjacent levels with preexisting degeneration was also uninvestigated.

In the present study, we established a FE model of the lumbosacral spine and simulated Pfirrman grade IV disc degeneration in L4-5[32]. Eight models were simulated, including the intact model, posterior decompression surgery (PD), PD with SIS stabilization and ligamentoplasty $(\mathrm{PD}+\mathrm{SIS})$, PD with SPI $(\mathrm{PD}+\mathrm{SPI})$, non-instrumented fusion with interbody cage implantation (Cage), semirigid lumbar fusion with cage implantation, SIS stabilization, and ligamentoplasty (Cage+SIS), semi-rigid lumbar fusion with cage and SPI (Cage+SPI) and instrumented PLIF with cage and pedicle screw instrumentation (Cage+PI). This study was aimed to evaluate the biomechanical property of semi-rigid stabilization and semi-rigid lumbar fusion. The range of motion (ROM) of the operated level were compared. We investigated the impact of different constructs on the adjacent levels simulated with preexisting degeneration. Critical parameters relevant to the development of ASD including ROM, intervertebral discs (IVD) stress, and strain energy were compared.

\section{Method}

Generation of lumbosacral FE model

A three-dimensional FE model of the lumbosacral spine from the third lumbar vertebra to the sacrum (L3-S5) was developed from the axial computed tomography $(\mathrm{CT})$ images at $1-\mathrm{mm}$ thickness $(512 \times 512)$ resolution, 16 -bit, and a pixel size of $0.3516 \mathrm{~mm} \times 0.3516 \mathrm{~mm}$, obtained from a resin spine model casted from a cadaveric spine. The CT images were imported into 3D-DOCTOR (Able Software Corp.) to construct the geometric structure of L3-Sacrum. The mesh was then prepared using Patran (MSC Software) and the FE model was imported into Abaqus 6.12 (Simulia Inc) to solve (Fig. 1). This study adopted linear and isotropic material properties for cortical bone, cancellous bone, posterior bony elements, 


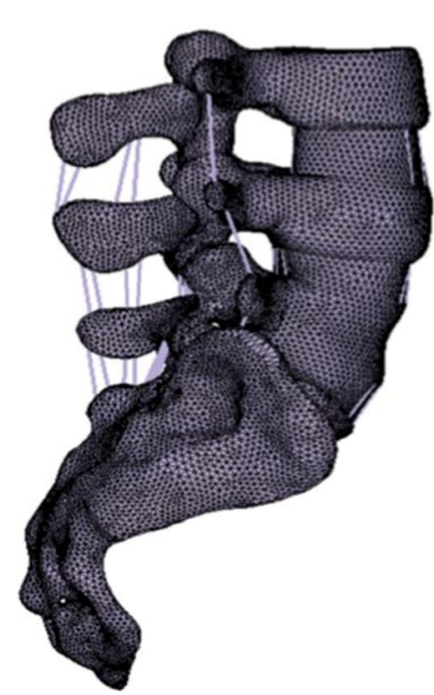

Intact
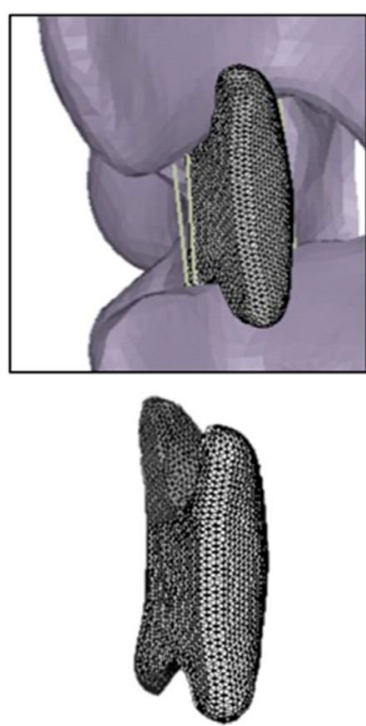

Interspinous

stabilizer
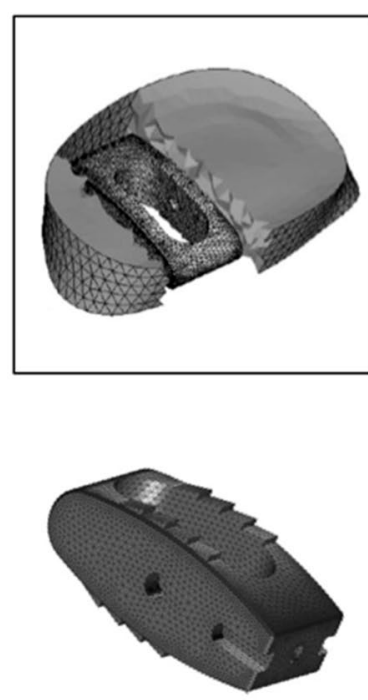

Cage

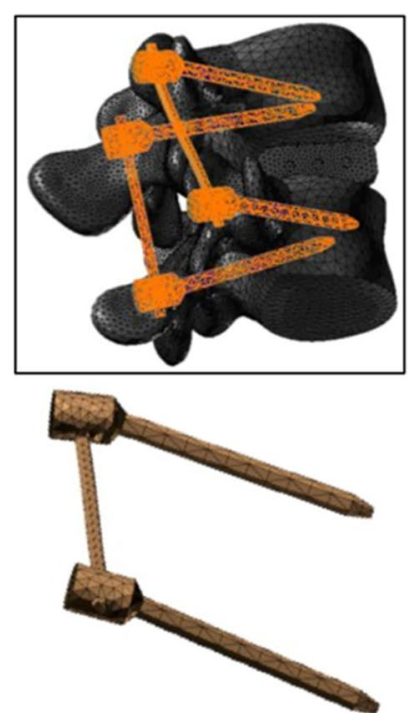

Pedicle screw and rod

Fig. 1 Finite element model of L3-Sacrum segments and surgical implants. The present finite element model of the intact spine (left), interspinous spacer (middle-left), interbody cage (middle-right) and pedicle screws and rods (right)

endplate, annulus fiber layers, annulus ground substance, and nucleus pulposus (Table 1).

A vertebra was composed of a cortical bone shell (thickness, $0.35 \mathrm{~mm}$ ), cancellous bone, endplates (thickness, $0.5 \mathrm{~mm}$ ), and posterior elements. A closed surface of cortical bone and endplates was assigned to 3-node S3R shell elements. Cancellous bone was assigned to 4-node C3D4 tetrahedral elements. The irregular posterior elements including the facet joints were modeled using C3D4 elements according to the original geometry. The contact of the facets was simulated with threedimensional surface-to-surface contact with friction and a finite sliding interaction was defined with the friction characteristic modeled with classic isotropic Coulomb friction model and a friction coefficient of 0.1 [33-35].

The FE model of the IVD consisted of annulus fiber layers, annulus ground substance and nucleus pulposus. The height of the discs at L3-4, L4-5, L5-S1 levels were 9.802, 7.65 , and $10.34 \mathrm{~mm}$, respectively. The model of the annulus fibrosus was created as ring-shaped by generating an outer border and an inner border using outer annulus fiber and inner annulus fiber, respectively. The superior and the inferior border of the annulus fibrosus were the adjacent endplates. The annulus fibers were modeled with six layers of shell elements with the thickness of each layer of $1.5 \mathrm{~mm}$. Annulus ground substance were defined between the two annulus fibers and the adjacent endplates and was modeled by C3D4 tetrahedral elements. Nucleus pulposus were defined by the inner annulus fiber and
Table 1 Material properties of the lumbosacral FE model and spinal implants

\begin{tabular}{lll}
\hline Component & Young's Modulus (MPa) & Poisson's Ratio \\
\hline Annulus Fiber & & \\
Inner Laminate: Inner Layer & 360 & 0.30 \\
Inner Laminate: Middle & 385 & 0.30 \\
Layer & & \\
Inner Laminate: Outer Layer & 420 & 0.30 \\
Outer Laminate: Inner Layer & 440 & 0.30 \\
Outer Laminate: Middle & 495 & 0.30 \\
Layer & & \\
Outer Laminate: Outer Layer & 550 & 0.30 \\
Annulus Ground Substance & 4.2 & 0.45 \\
Nucleus Pulposus & 1 & 0.49 \\
Cancellous Bone & 100 & 0.20 \\
Cortical Bone & 12,000 & 0.30 \\
Posterior Bony Elements & 3500 & 0.25 \\
Endplate & 12,000 & 0.30 \\
ALL/PLL/LF/ISL/SSL & $20 / 20 / 20 / 10 / 10$ & 0.25 \\
DIAM core & 20 & 0.45 \\
DIAM Truss & 5000 & 0.20 \\
Graft Bone & 100 & 0.20 \\
PEEK & 3600 & 0.25 \\
Titanium screw/rod & 110,000 & 0.30 \\
\hline
\end{tabular}


adjacent endplates and was modeled by C3D4elements. In the present study, Pfirrmann grade IV moderate degeneration in L4-5 and Pfirrmann grade III low grade degeneration in the adjacent L3-4 and L5-S1 IVDs were simulated. In Pfirrmann grade III degeneration, the disc height was preserved [32, 36]. However, the annulus degenerated and previous study reported a decrease of annulus fibrosus elastic modulus in the mildly to moderately degenerated IVD [37, 38]. With this in mind, the degenerated IVD was simulated by modifying the material properties of the annulus fibrosus with the elastic moduli of the annulus fiber layers reduced by $80 \%$ in L4-5 to simulate moderately degenerated IVD and 20\% in L3-4, L5-S1 to simulate mildly degenerated adjacent levels [39].

Ligamentous complex including anterior longitudinal ligaments (ALL), posterior longitudinal ligaments (PLL), ligamentum flavum (LF), interspinous ligaments (ISL) and supraspinous ligaments (SSL) were modeled using hyperelastic, tension-only, Truss elements (T3D2). The properties of the ligaments were adopted from Goel et al. [40] and given in Table 2. The element types and number of elements used in the components of the spine are listed in Table 3.

\section{Generation of the implant models}

The implant model for the SIS was generated according to the DIAM interspinous (Medtronic) implant. The primary dimensions (width, length, heights) were

Table 2 Properties of the ligaments in the present study

\begin{tabular}{llllll}
\hline Ligament & ALL & PLL & LF & ISL & SSL \\
\hline Elastic modulus (small strain)(MPa) & 7.8 & 10 & 15 & 10 & 8 \\
Transition strain (\%) & 12 & 11 & 6.2 & 14 & 20 \\
Elastic modulus (large strain)(MPa) & 20 & 20 & 19.5 & 11.6 & 15 \\
Cross sectional area (mm2) & 52.8 & 16 & 66.8 & 26 & 23 \\
\hline
\end{tabular}

Table 3 Element type and number of element in the intact lumbosacral FE model

\begin{tabular}{|c|c|c|c|c|c|c|}
\hline \multirow[t]{2}{*}{ Component } & \multirow[t]{2}{*}{ Element type } & \multicolumn{5}{|c|}{ No. of Elements } \\
\hline & & L3 & L4 & L5 & Sacrum & \\
\hline Cortical Bone & S3R & 1334 & 1913 & 2027 & 30,212 & \\
\hline Cancellous Bone & C3D4 & 18,166 & 20,129 & 22,756 & 131,828 & \\
\hline Endplate & S3R & 1833 & 2013 & 2193 & 1615 & \\
\hline \multirow[t]{2}{*}{ Posterior Bony Elements } & C3D4 & 26,695 & 27,739 & 25,313 & & \\
\hline & & L3-L4 & L4-L5 & L5-S1 & & \\
\hline Nucleus Pulposus & C3D4 & 4942 & 3998 & 5670 & & \\
\hline Annulus Ground Substance & C3D4 & 8262 & 4619 & 7063 & & \\
\hline Annulus Fiber & STRI3 & 1963 & 1143 & 1350 & & \\
\hline Ligament & & ALL & PLL & LF & ISL & SSL \\
\hline No. of Elements & T3D2 & 12 & 12 & 12 & 6 & 6 \\
\hline
\end{tabular}

$14 \mathrm{~mm}, 28 \mathrm{~mm}$, and $12 \mathrm{~mm}$ respectively, and the interspinous distance of the implant body in between the two wings was $12 \mathrm{~mm}$. The interspinous implant composed of a silicon core covered inside a polyester mesh, which connects to a pair of polyester mesh tethers that ties the spacer on to the adjacent spinous processes for ligamentoplasty. The three-dimensional structure of the interspinous spacer was created in software Patran (MSC Software Corp.) and mesh structures were prepared using software Hypermesh 11.0 (Altair Technologies Inc) (Fig. 1). The implant body composed of C3D4 solid elements, and the tethers were assigned to tension-only Truss element. The contact surface between the spinous process and the interspinous spacer was assumed with a coefficient of friction of 0.5.

For interbody fusion, the model for lumbar interbody cage was developed from the CAPSTONE ${ }^{\circledR}$ PEEK Spinal System (medtronic) using Patran software (MSC Software Corp.). Mesh structures were prepared and assigned to C3D4 solid elements using software Hypermesh 11.0(Altair Technologies Inc). The cage composed of polyetheretherketone (PEEK) and the primary dimensions (width, length, heights) were $14 \mathrm{~mm}, 26 \mathrm{~mm}$ and $10 \mathrm{~mm}$, respectively (Fig. 1). The primary dimensions (diameter, length) of the pedicle screws for lumbar posterior instrumentation were $5.5 \mathrm{~mm} \times 45 \mathrm{~mm}$ and $6.5 \mathrm{~mm} \times 45 \mathrm{~mm}$, respectively. The diameter of the rods was $6.0 \mathrm{~mm}$ (Fig. 1). The pedicle screws and the rigid rods were composed of titanium. The semi-rigid rods were composed of PEEK. The material properties and element types assigned to the implants were shown in Table 1.

\section{Simulation of surgeries}

Eight models were simulated, including the intact model, posterior decompression surgery (PD), PD with SIS stabilization and ligamentoplasty (PD+SIS), PD with SPI 
$(\mathrm{PD}+\mathrm{SPI})$, non-instrumented fusion with interbody cage implantation (Cage), semi-rigid lumbar fusion with cage implantation, SIS stabilization, and ligamentoplasty (Cage+SIS), semi-rigid lumbar fusion with cage and SPI (Cage+SPI) and instrumented PLIF with cage and pedicle screw instrumentation (Cage+PI) (Fig. 2).

For L4-5 posterior decompression, a unilateral interlaminar decompression with medial facetectomy was simulated by removing the inferior half of the L4 laminar, the superior one-fourth of the L5 laminar and the medial twenty-percent of the L4-5 articular facets. Interbody cage fusion over L4-5 was performed with cage placement after total removal of the nucleus and partial removal of the PLL and posterior annulus.

\section{Loading and boundary conditions}

The boundary conditions imposed in this study for all models were set with the nodes on the sacroiliac joint surface constrained in all directions. The facet interaction and the spinous process-interspinous spacer interaction were defined as finite sliding, surface-to-surface contact model with classical Coulomb friction. The endplate-cage boundaries were defined as tie-constraint.

A preload of $150 \mathrm{~N}$ was applied evenly on the superior endplate of the L3 vertebral body using follower load technique to stimulate the axial loading of the lumbosacral spine. For simulations of lumbosacral motions including flexion, extension, lateral bending, and axial rotation, a $3 \mathrm{~N}-\mathrm{m}$ moment was applied evenly on the L3-L5 segments, with a moment of $1 \mathrm{~N}$-m on each segment. The rationale behind the evenly distributed moment application is that the primary muscles producing lumbar spine motions such as the psoas muscle (flexion), erector spinae and semispinalis (extension), quadratus lumborum and external/internal obliques (lateral bending), semispinalis, and deep posterior paraspinal muscles (rotation) all had multiple attachments on multiple lumbar segments. As a result, an evenly distributed moment could represent a more physiological condition.

\section{Convergence test}

We used the intact model for convergence test and measured the displacement of a reference point on the center of the L3 superior endplate under a $150 \mathrm{~N}$ preload. Four different amounts: 516783, 509,525, 354,824, and 272,588 elements were compared for their corresponding displacements using. 516,783 elements as reference. The final FE model consisted of 354,824 elements connected through 69,134 nodes. The relative displacement error was less than $3.75 \%$ and the element size ranged from 1.0 to $2.0 \mathrm{~mm}$.

\section{Results \\ Model validation}

For the validation of the FE model, we compared the simulated ROM and IVD stress of the intact model with those reported in the literature (Fig. 3). First, the ROMs of the present intact FE model of the lumbosacral spine were compared with the in vivo measurements and in vitro experiments conducted by Pearcy et al., White et al., and Yamamoto et al. [41-44]. The average segmental ROMs of the present intact model were as follows: flexion-extension, 8.85; lateral bending, 2.31; and axial rotation, 2.22 (Degrees). The ROMs were in good agreements with the literature.

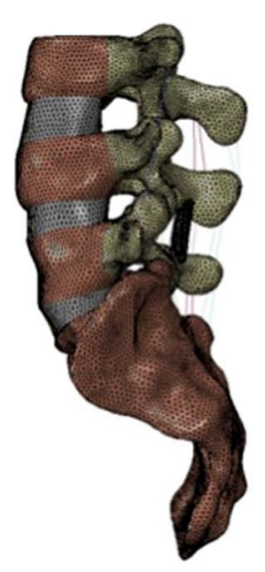

PD+SIS

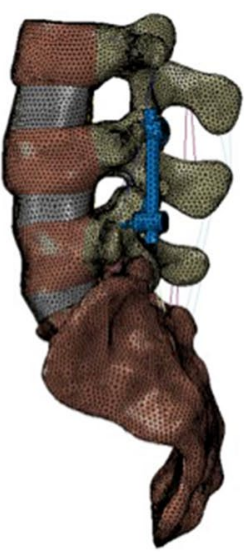

PD+SPI

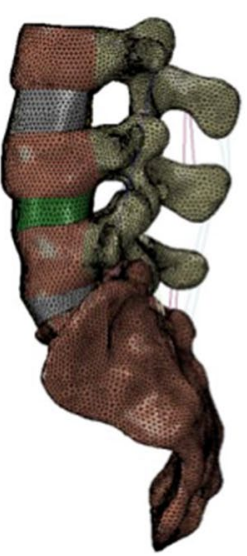

Cage

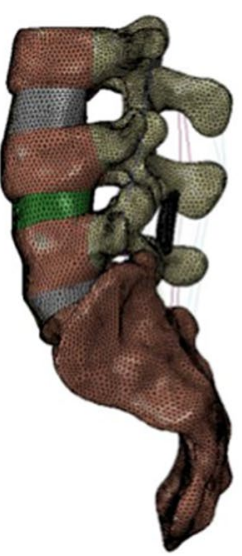

Cage + SIS

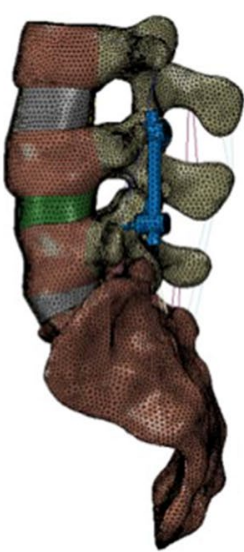

Cage+SPI

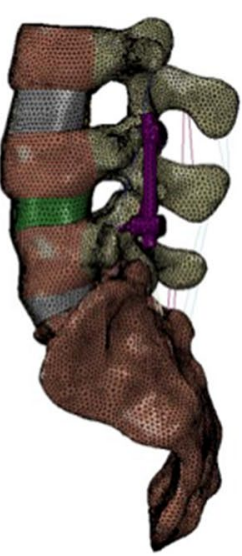

Cage+PI

Fig. 2 Different L4/5 surgical constructs. Surgical constructs simulated in this study including PD + SIS, PD + PSI, Cage, Cage+SIS, Cage+SPI, and Cage+PI. Interbody fusion with cage implantation was indicated in green. Semi-rigid and rigid instrumentation were indicated in blue and purple, respectively 

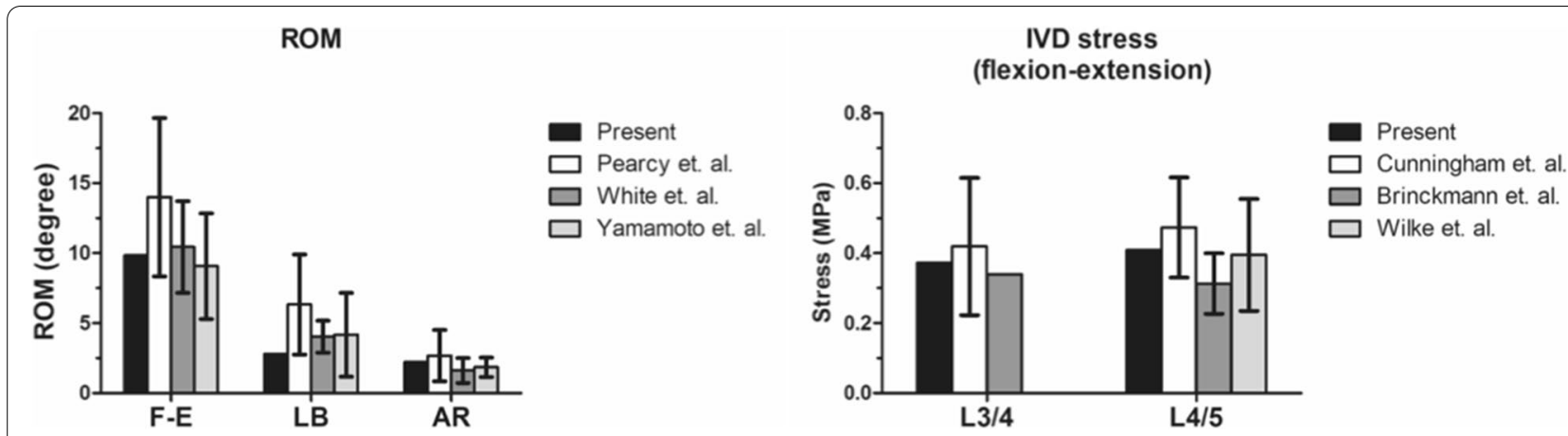

Fig. 3 Validation of the present finite element model. Comparisons between the ROM and IVD stress of the present intact model with the literature (presented in mean and standard deviation)

Second, the model was validated with the in vitro measurements of lumbar intradiscal pressure conducted by Cunningham et al., Brinckmann et al. and Wilke et al. [45-47]. In the previous in vitro experiments, the average stress in the IVD under sagittal flexion-extension ranged from $0.22 \mathrm{Mpa}$ to $0.61 \mathrm{MPa}$ in $\mathrm{L} 3-4$ and $0.23 \mathrm{MPa}$ to $0.62 \mathrm{MPa}$ in L45. The IVD stress under flexion-extension in the present model were $0.37 \mathrm{Mpa}$ in $\mathrm{L} 3-4$ and $0.41 \mathrm{MPa}$ in L4-5, which were compatible with the in vitro results (Fig. 3).

\section{ROM at the operated level}

The ROM of each construct under flexion, extension, lateral bending and axial rotation were shown in Fig. 4. Compared to the intact model, PD resulted in hypermobility at the operated level under all motions, which is accompanied by a marked increase in ROM in the suprajacent level (L3-4) during lateral bending and a mild increase in ROM in the infrajacent level (L5-S1) during flexion, extension and lateral bending. The application of semi-rigid stabilization with SIS after PD (PD+SIS) decreased the ROM of the decompressed level. Compared to PD + SIS, PD + SPI had more ROM reduction at the operated level in all motion, especially in lateral bending.

Comparison between the constructs intended to achieve fusion including Cage, Cage+SIS, Cage+SPI and Cage+PI revealed that all fusion constructs reduced the ROM at the operaed level. In flexion, Cage+PI had the most ROM reduction, while Cage only had the least ROM reduction. The difference between Cage+SIS and Cage+SPI was less than 0.03 degree. In extension, while Cage had the least ROM reduction, the differences between Cage+SIS, Cage+SPI, and Cage+PI were less than 0.05 degree. In lateral bending, Cage+PI had better ROM reduction than other three constructs. In axial rotation, pedicle screw-based constructs (Cage+SPI and
Cage+PI) had significant more ROM reduction compared to Cage and Cage+SIS. The difference between Cage+SPI and Cage+PI was less than 0.02 degree in axial rotation. In summary, the ROM reduction of semirigid fusion constructed were better than non-instrumented fusion. Comparing Cage+SIS and Cage + SPI instrumented fusion (Cage $+\mathrm{PI}$ ), the result showed that the three fusion strategies had comparable performance in $\mathrm{ROM}$ reduction in the operated level in flexion and extension, but Cage+PI had a better ROM reduction in lateral bending. The major difference of Cage+SIS and Cage+SPI was observed in axial rotation with Cage+SPI had better ROM reduction than Cage+SIS. The values of the ROM were shown in Table 4.

\section{ROM at the adjacent levels}

Semi-rigid stabilization with SIS or SPI after PD resulted ROMs similar to that of the intact spine. Moreover, the significant increase in lateral bending ROM after PD was alleviated in PD+SIS and PD + SPI (Fig. 4). Comparison between the fusion models showed that all four fusion constructs had comparable ROM to the intact spine in the suprajacent level(L3-4) in flexion, extension, and axial rotation. In lateral bending, while Cage+SPI reduced the ROM at the suprajacent level by $13 \%$, a 17,18 , and 9\% increased motion in lateral bending in the suprajacent level was noted for Cage, Cage+SIS, and Cage+PI, respectively.

For the infrajacent level, pedicle screw-based constructs resulted in significant hypermobility of all motions with 24.1, 27,7, 25.9, and 10.3\% ROM increase for Cage+SPI and 26.1, 30.0, 27.1, and 10.8\% ROM increase for Cage $+\mathrm{PI}$ in flexion, extension, lateral bending and axial rotation respectively. In contrast, Cage and Cage+SIS only increased the motion at the infrajacent level by 9.3 and 3.6\% in flexion, 5.3 and $2.8 \%$ in extension, 10.5 and $11.2 \%$ in lateral bending, respectively. Moreover, 


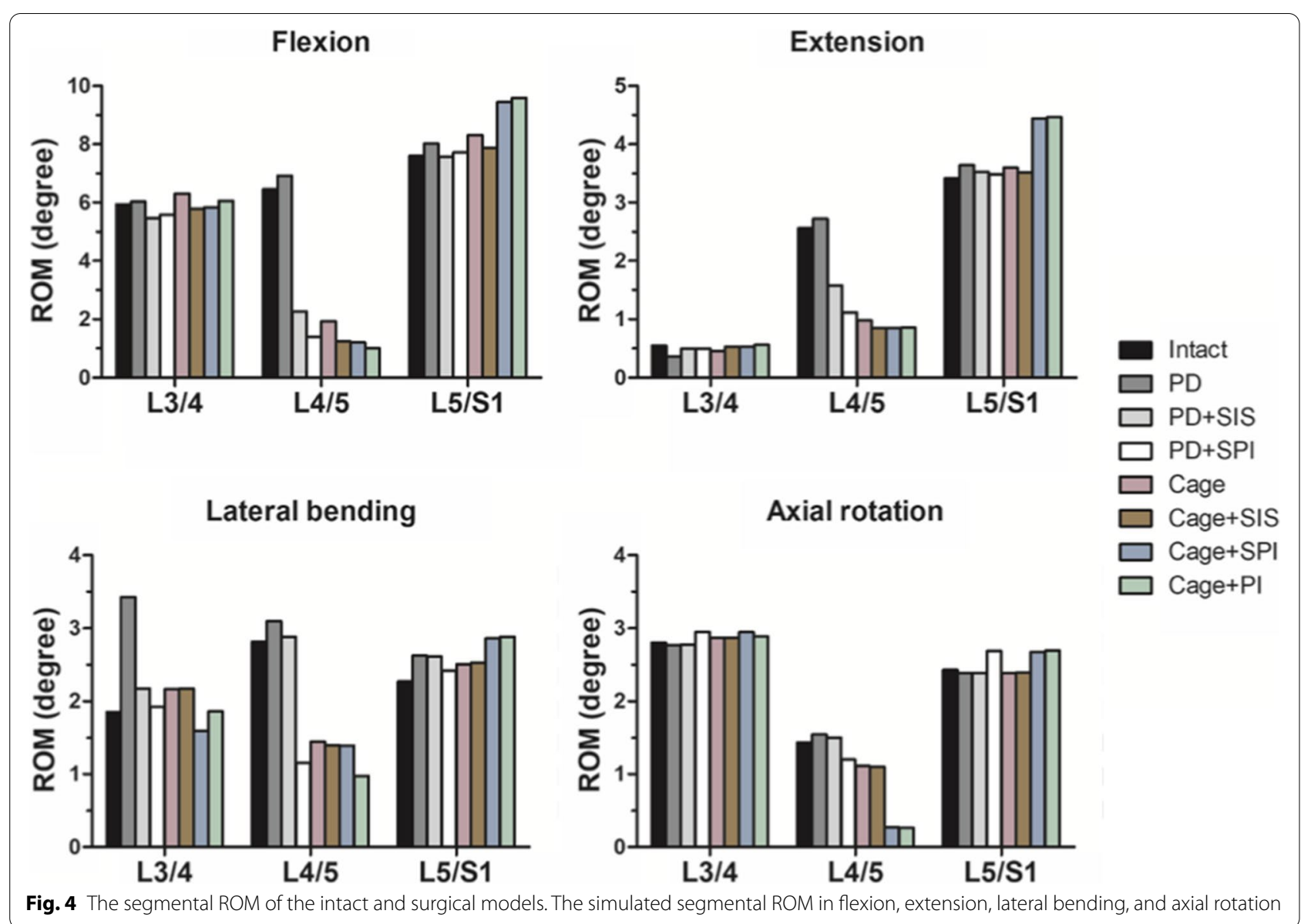

Table 4 ROM of the intact and surgical models

\begin{tabular}{|c|c|c|c|c|c|c|c|c|}
\hline Flexion & Intact & PD & $\mathrm{PD}+\mathrm{SIS}$ & $\mathrm{PD}+\mathrm{SPI}$ & Cage & Cage+SIS & Cage + SPI & Cage $+\mathrm{PI}$ \\
\hline $\mathrm{L} 3 / 4$ & 5.94 & 6.03 & 5.47 & 5.58 & 6.30 & 5.78 & 5.83 & 6.05 \\
\hline$\llcorner 4 / 5$ & 6.45 & 6.93 & 2.27 & 1.40 & 1.93 & 1.24 & 1.21 & 1.01 \\
\hline$L 5 / S 1$ & 7.60 & 8.02 & 7.57 & 7.72 & 8.31 & 7.88 & 9.45 & 9.59 \\
\hline Extension & Intact & PD & $\mathrm{PD}+\mathrm{SIS}$ & $\mathrm{PD}+\mathrm{SPI}$ & Cage & Cage+SIS & Cage+SPI & Cage+PI \\
\hline$\llcorner 3 / 4$ & 0.55 & 0.36 & 0.50 & 0.51 & 0.45 & 0.53 & 0.53 & 0.56 \\
\hline$\llcorner 4 / 5$ & 2.56 & 2.72 & 1.58 & 1.12 & 0.98 & 0.85 & 0.86 & 0.88 \\
\hline$L 5 / S 1$ & 3.42 & 3.64 & 3.53 & 3.49 & 3.6 & 3.52 & 4.45 & 4.46 \\
\hline Lateral bending & Intact & PD & $\mathrm{PD}+\mathrm{SIS}$ & $\mathrm{PD}+\mathrm{SPI}$ & Cage & Cage+SIS & Cage+SPI & Cage+PI \\
\hline $\mathrm{L} 3 / 4$ & 1.85 & 3.43 & 2.18 & 1.92 & 2.17 & 2.18 & 1.60 & 1.86 \\
\hline$\llcorner 4 / 5$ & 2.82 & 3.11 & 2.88 & 1.15 & 1.45 & 1.40 & 1.39 & 0.98 \\
\hline L5/S1 & 2.27 & 2.63 & 2.62 & 2.42 & 2.51 & 2.53 & 2.86 & 2.89 \\
\hline Axial rotation & Intact & PD & $P D+S I S$ & $\mathrm{PD}+\mathrm{SPI}$ & Cage & Cage+SIS & Cage+SPI & Cage+PI \\
\hline $\mathrm{L} 3 / 4$ & 2.80 & 2.77 & 2.78 & 2.95 & 2.87 & 2.87 & 2.95 & 2.89 \\
\hline$\llcorner 4 / 5$ & 1.44 & 1.55 & 1.51 & 1.23 & 1.15 & 1.12 & 0.27 & 0.26 \\
\hline L5/S1 & 2.43 & 2.39 & 2.39 & 2.69 & 2.39 & 2.42 & 2.68 & 2.69 \\
\hline
\end{tabular}

Cage and Cage+SIS reduced the ROM by 1.8 and $1.5 \%$ in axial rotation compared to the intact model. The values of the ROM were shown in Table 4.
The von Mises stress and strain energy at the operated level

The maximal von Mises stress of the intervertebral 
disc or the interbody cage at the operated level (L4-5) of each construct were shown in Fig. 5. All constructs decreased the maximal von Mises stress at the operated level (L4-5) in all motions. In flexion, Cage, Cage+SIS, Cage + SPI, and Cage + PI reduced the stress at the fusion level by $10.3 .0,18.2,25.8$, and $32.4 \%$ compared to the intact model, respectively. In extension, Cage, Cage+SIS, Cage+SPI, and Cage+PI reduced the stress by 38.7, 43.4, 73.3 , and $75.7 \%$, respectively. In lateral bending, the differences of the stress in the implanted cage were less than 4.3\%. In axial rotation, higherstress of the interbody cage was found in Cage+SPI and Cage+PI while Cage and Cage+SIS had less stress.

The strain energy in the L4-5 intervertebral disc was comparable among non-fusion constructs in flexion, lateral bending, and axial rotation. In extension, PD resulted in an increased strain energy at the operated level, which can be alleviated by semi-rigid stabilization. (Fig. 6). All fusion constructs decreased the strain energy of the operated level in extension, lateral bending, and axial rotation. The main difference in strain energy reduction was noted in flexion and extension. In flexion, Cage+SPI and Cage+PI reduced the L4/5 strain energy markedly by 79.1 and $80.8 \%$, respectively, but Cage+SIS only had $15.9 \%$ decrease. Moreover, fusion with interbody cage only (Cage) was unable to reduce the L4/5strain energy.

\section{The von Mises stress and strain energy at the adjacent intervertebral disc}

Compared to the intact model, all non-fusion constructs had decreased IVD stress at both adjacent levels in all motions. For fusion constructs, Cage + SPI and Cage + PI resulted in increased von Mises stress at both adjacent levels in all motions compared to Cage and Cage+SIS. In contrast, Cage, and Cage+SIS had decreased von Mises stress compared to the intact model in all motions. The average percentage of decrease in the von Mises stress at the adjacent levels compared to the intact model was $8.7 \%$ and $9.0 \%$ for Cage and Cage+SIS, respectively.

In terms of the strain energy of the adjacent IVDs, the difference of the strain energy at the suprajacent level among all models was less than $7.9 \%$ (Fig. 6). In contrast, compared to the intact model, the strain energy at the infrajacent level under extension, lateral bending, and

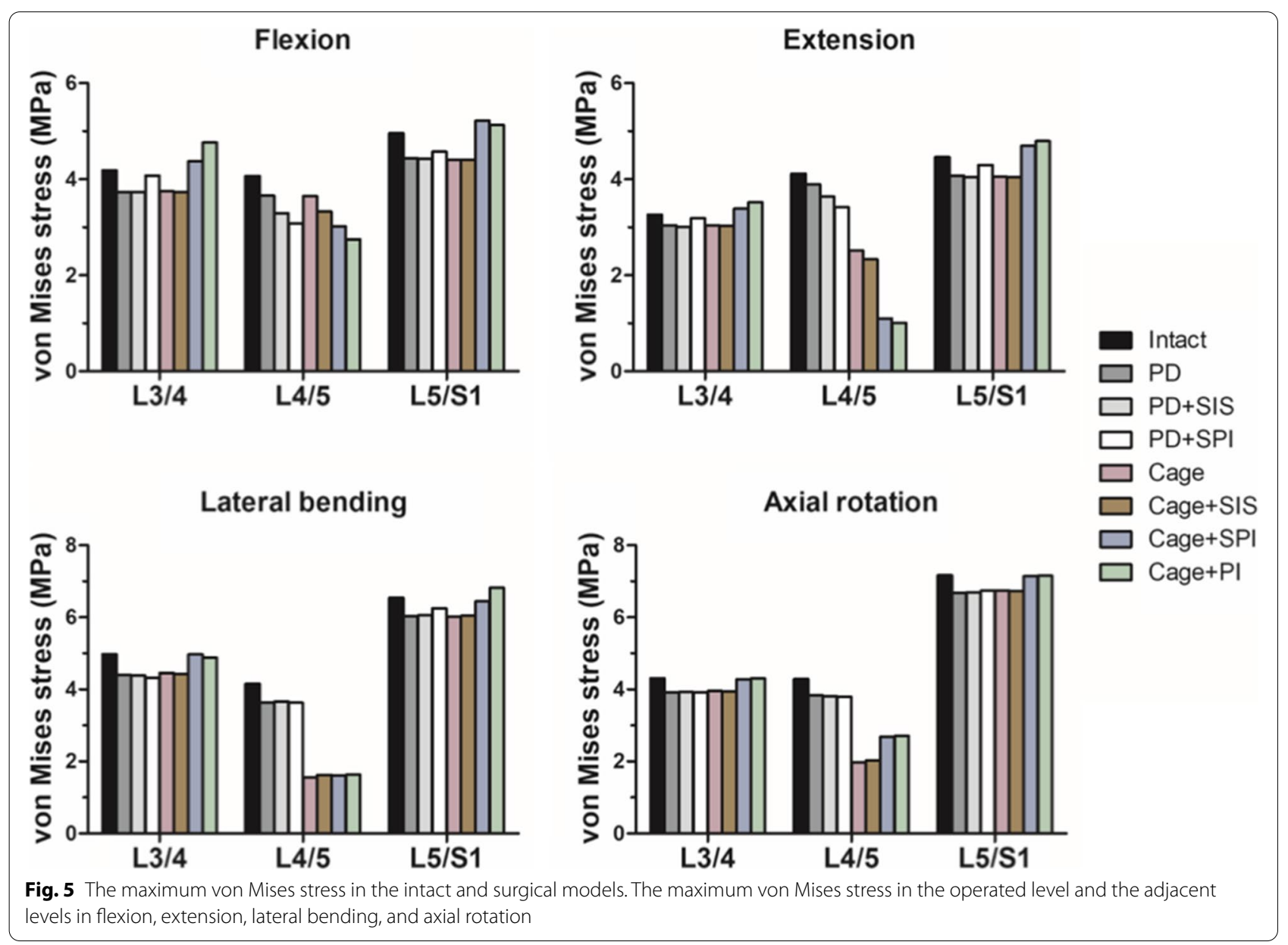




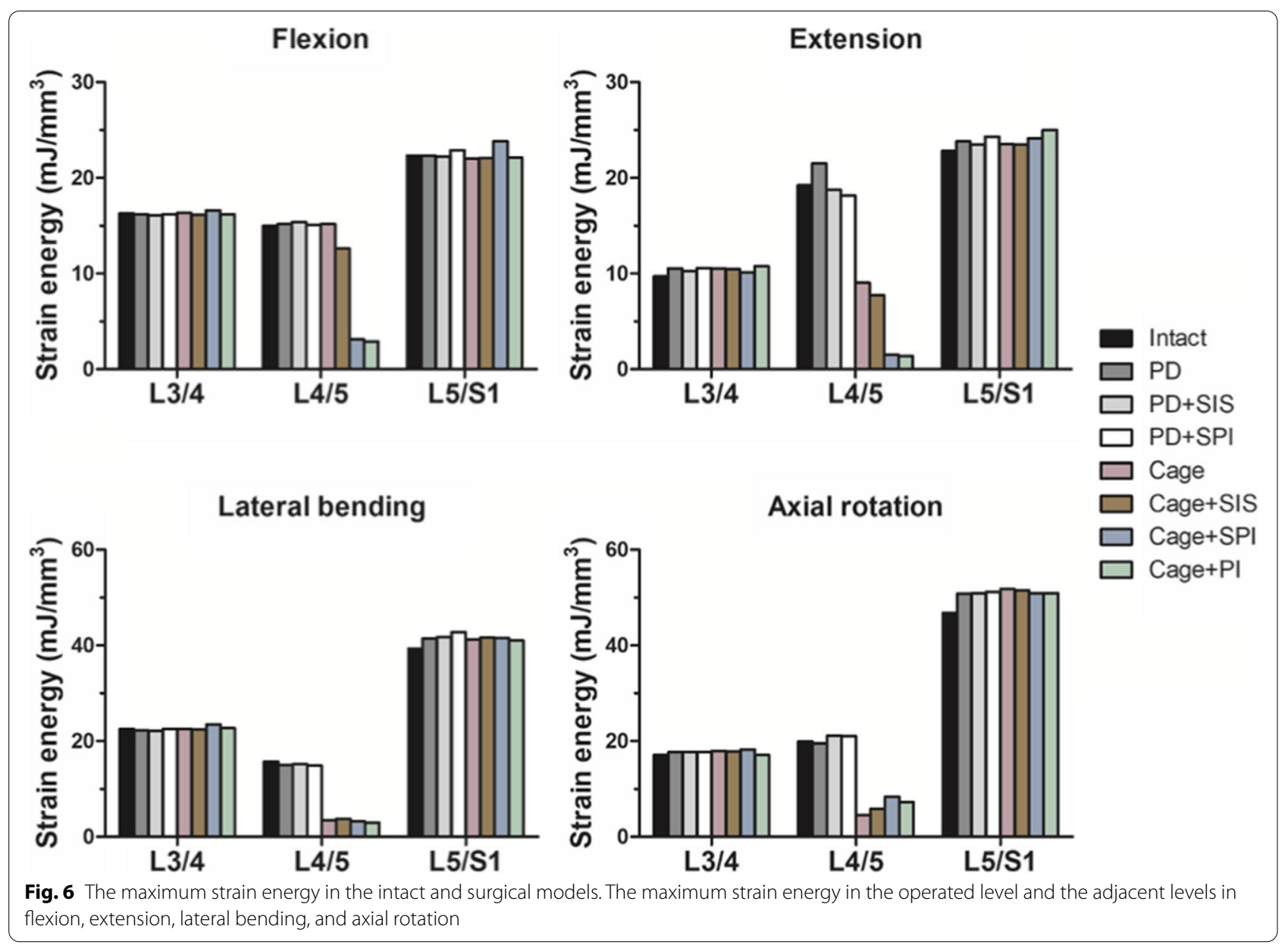

axial rotation was increased in allsurgical constructs, but the percentages of increase were all less than $9 \%$.

\section{Discussion}

To investigate the impact of the semi-rigid fusion constructs on the fusion and the adjacent levels, the present study utilized FE model to simulate semi-rigid non-fusion and fusion constructs involving SIS and SPI and compared them to rigid PI constructs. We showed Cage+SIS, and Cage+SPI provided similar stabilization in flexion-extension and lateral bending of the fusion level compared to Cage+PI but Cage+SIS was less resistant to axial rotation. Our work revealed Cage+SIS had advantages in less increase of infradjacent ROM and reduced adjacent IVD stresses compared to SPI and PI. The difference in strain energy between the constructs was less than $7.9 \%$, but all constructs increased the strain energy in the infradjacent level.

In the current FE model, we simulated preexisting disc degeneration on both adjacent levels and compared critical parameters relevant to the development of ASD such as ROM, IVD stress, and strain energy. Preexisting disc degeneration is a major risk factor for ASD[48]. Since we aimed to evaluate the impact of the constructs on the adjacent levels, the assumption of preexisting degeneration is more clinically relevant compared to simulation with intact discs. The present model was validated with previously published in vitro experiments. The simulated ROM and IVD stress were compatible with the cadaveric results in the literature [38-44]. Since lumbosacral disc degeneration is most prevalent at L4/5 followed by L5-S1 and L3-4 $[49,50]$, we chose L4/5 as the operated level and aimed to optimize the lumbar constructs in order to prevent ASD especially at the adjacent level after lumbar interbody fusion.

Pseudarthrosis is a well-known complication of lumbar fusion. To prevent pseudarthrosis, sufficient stabilization to minimize the motion at the fusion level is of paramount importance[51]. Previous study evaluated SIS device in PLIF constructs and suggested that the SIS device and PI performed equivalently in flexion-extension, but the pedicle screws construct was more resistant to lateral bending[29]. Our simulation showed that semi-rigid 
lumbar fusion Cage+SIS and Cage+SPI had comparable ROM reduction to rigid PI (Cage+PI) at the fusion level under flexion-extension. In lateral bending and axial rotation, only Cage + SPI had comparable ROM reduction to Cage+PI. Together, these results suggested SIS-based lumbar fusion had better stabilization in flexion-extension but was less resistant to lateral bending and rotation. Since excessive motion at the level intended to fuse may hinder bony fusion and was associated non-union [52], further clinical study is needed to investigate whether additional maneuvers such as application of lumbar bracing to limit lateral axial rotation may be helpful to provide support and facilitate fusion after a semi-rigid lumbar fusion with SIS. However, it should be noted that since the primary motion of the lumbar spine is flexion-extension [53], the semi-rigid constructs provided sufficient stabilization in the primary lumbar motion.

Hypermobility in the adjacent levels is a significant risk factor of $\mathrm{ASD}$ [54], and preservation of lumbar motion was associated with a lower incidence of lumbar ASD[55]. A construct causing less adjacent hypermobility might help prevent adjacent degeneration and therefore we investigated the adjacent ROM in the semi-rigid constructs. Compared to Cage+SPI and Cage+PI, which caused hypermobility at the infradjacent level, Cage+SIS resulted in a minimal impact in terms of ROM at L5-S1. Our results suggested that semi-rigid lumbar fusion has an advantage in prevention of L5-S1 ASD.

Acute material failure occurs when von Mises stress surpasses the tensile yield stress [56]. For intervertebral discs, it might clinically present as an acutely ruptured annulus with disc herniation compressing the thecal sac or nerve root [57]. Our results revealed that while pedicle screwbased construct (Cage+SPI and Cage $+\mathrm{PI}$ ) increased the von Mises stress in adjacent discs, Cage+SIS decreased the von Mises stress in adjacent discs. Another clinically relevant parameter is the strain energy at the adjacent intervertebral disc. The strain energy stored in the material during repetitive motion is related to fatigue of the material and this may make the IVD susceptible to chronic disc degeneration and progression of the pre-existing disc degeneration to higher-grade degeneration [58]. An increased strain energy at the infrajacent was found among all surgical constructs in extension, lateral bending, and axial rotation compared to the intact model. Although the percentages of increase were less than $9 \%$, it may imply that all L4/5 surgical constructs may increase the susceptibility of L5S1 to chronic degeneration.

Despite that, the comparisons of von Mises stress and strain energy between Cage+SIS, Cage + SPI and Cage + PI still revealed that Cage+SIS decreased the von Mises stress at adjacent levels and had less increase in strain energy at L5-S1 in flexion-extension. This may suggest
Cage +SIS had a pontential advantage in lower risks of ASD. Taken together, our results suggested that Cage+SIS and Cage+SPI can provide sufficient stabilization and ROM reduction to facilitate spinal fusion. In addition, our results showed that semi-rigid lumbar fusion with SIS had less impact on adjacent levels in terms of less hypermobility and less von Mises stress. However, Cage+SIS had a disadvantage of less ROM reduction in lateral bending and axial rotation. Further clinical studies are warranted to investigate whether additional maneuvers such as lumbar bracing should be applied to limit the motions and minimize the disacvantage of Cage+SIS.

The limitations of the present study should be noted. First, the IVD and the bony tissue was modeled using linear isotropic material properties and the anisotropic properties of the materials were neglected. Second, hyperelastic Truss elements were used to simulate the ligaments, which neglected the contact interaction between ligaments and vertebrae but also avoided the unrealistic shearing forces in the ligaments. The simplifications reduced the computation time. Other limitations included that the position and trajectory of the screws and interspinous implants were likely to have variations. Changes in orientation of the implants may alter the biomechanics of the construct but this is very challenging to be incorporated into the simulation since multiple real-world factors such as surgical approach, anatomical variation, and surgeon's preference all affect the positioning of the implant. Last, perfect surface contact with tie constraints were made between pedicle screws, cage, and bone. This assumption was an advantage over cadaveric experiments since it allowed us to simulate spinal adaptation to bony fusion [59]. Despite these limitations, the main conclusions of the present study are based on comparisons between the surgical models. The above-mentioned model simplifications are applied to all models and likely have minimal effect on the comparative differences.

\section{Conclusion}

Semi-rigid fusion constructs can provide adequate stabilization and flexion-extension ROM reduction at the fusion level similar to rigid fixation in flexion-extension. In addition, SIS-assisted fusion caused less hypermobility and less von Mises stress in the adjacent levels. However, SIS-assisted fusion had a disadvantage of less ROM reduction in lateral bending and axial rotation. Further clinical studies are warranted to investigate whether additional maneuvers such as lumbar bracing should be applied to minimize the disadvantage of SIS-assisted fusion and to evaluate the clinical efficacy and safety of semi-rigid fusions. 


\section{Abbreviations}

SIS: Semi-rigid interspinous sstabilization; SPI: Semi-rigid posterior instrumentation; PEEK: Polyetheretherketone; ROM: Range of motion; PLIF: Posterior lumbar interbody fusion; PI: Posterior instrumentation; ASD: Adjacent segment degeneration; IVD: Intervertebral disc; ALL: Anterior longitudinal ligaments; PLL: Posterior longitudinal ligaments; LF: Ligamentum flavum; ISL: Interspinous ligaments; SSL: Supraspinous ligaments; PD: Posterior decompression.

\section{Acknowledgements}

Not applicable.

\section{Authors' contributions}

C-EW and K-YH: conceptualization; C-EW, H-TH, L-SK, C-JL, K-CC and K-YH: methodology; L-SK, C-JL, K-CC and H-TH: software; C-EW: validation; C-EW and $\mathrm{K}-\mathrm{YH}$ : formal analysis; $\mathrm{C}-\mathrm{EW}$ and $\mathrm{K}-\mathrm{YH}$ : investigation; $\mathrm{H}-\mathrm{TH}$ and $\mathrm{K}-\mathrm{YH}$ : resources; C-EW, L-SK, C-JL and K-CC: data curation; C-EW and K-YH: writing - original draft preparation; C-EW, H-TH, L-SK, C-JL, K-CC and K-YH: writing - review and editing; C-EW, L-SK and C-JL: visualization; K-YH: supervision. All authors have read and approved the final manuscript.

\section{Authors' information}

Not applicable.

\section{Funding}

This study was performed, in part, with support from Ministry of Science and Technology of Taiwan (MOST 108-2314-B-006-048-MY2; MOST 110-2314-B006-020). The funding agency had no role in study design, data collections, analysis and interpretation of data, writing of the manuscript, and decision to submit the manuscript for publication.

\section{Availability of data and materials}

The datasets used and/or analysed during the current study are available from the corresponding author on reasonable request.

\section{Declarations}

\section{Ethics approval and consent to participate}

Not applicable.

\section{Consent for publication}

Not applicable.

\section{Competing interests}

The authors declare that they have no competing interests.

\section{Author details}

'Section of Neurosurgery, Department of Surgery, National Cheng Kung University Hospital, College of Medicine, National Cheng Kung University, Tainan, Taiwan. ${ }^{2}$ Department of Civil Engineering, National Cheng Kung University, Tainan, Taiwan. ${ }^{3}$ Department of Civil and Disaster Prevention Engineering, National United University, Miaoli, Taiwan. ${ }^{4}$ Department of Orthopedics, National Cheng Kung University Hospital, College of Medicine, National Cheng Kung University, Tainan, Taiwan.

Received: 18 January 2021 Accepted: 12 December 2021

Published online: 03 January 2022

\section{References}

1. Lin PM. Posterior lumbar interbody fusion technique: complications and pitfalls. Clin Orthop Relat Res. 1985;:90-102. http://www.ncbi.nlm.nih. gov/pubmed/3882302.

2. Cloward RB. Posterior lumbar interbody fusion updated. Clin Orthop Relat Res. 1985;:16-9. http://www.ncbi.nlm.nih.gov/pubmed/3971616.

3. Prolo DJ, Oklund SA, Butcher M. Toward uniformity in evaluating results of lumbar spine operations. A paradigm applied to posterior lumbar interbody fusions. Spine (Phila Pa 1976). 11:601-6. doi:https://doi.org/10. 1097/00007632-198607000-00012.
4. YeY Chen $\mathrm{D}, \mathrm{XuH}$. The comparison of instrumented and noninstrumented fusion in the treatment of lumbar spondylolisthesis: a meta-analysis. Eur Spine J. 2014;23:1918-26. https://doi.org/10.1007/ s00586-014-3453-1

5. Babu MA, Coumans J-VC, Carter BS, Taylor WR, Kasper EM, Roitberg BZ, et al. A review of lumbar spinal instrumentation: evidence and controversy. J Neurol Neurosurg Psychiatry. 2011;82:948-51. https://doi.org/10. 1136/jnnp.2010.231860

6. Park P, Garton HJ, Gala VC, Hoff JT, McGillicuddy JE. Adjacent Segment Disease after Lumbar or Lumbosacral Fusion: Review of the Literature. Spine (Phila Pa 1976). 2004;29:1938-44. https://doi.org/10.1097/01.brs. 0000137069.88904 .03 .

7. Eck JC, Humphreys SC, Hodges SD. Adjacent-segment degeneration after lumbar fusion: a review of clinical, biomechanical, and radiologic studies. Am J Orthop (Belle Mead NJ). 1999;28:336-40 http://www.ncbi.nlm.nih. gov/pubmed/10401898.

8. Jain P, Rana M, Biswas JK, Khan MR. Biomechanics of spinal implants-a review. Biomed Phys Eng Express. 2020;6:042002. https://doi.org/10.1088/ 2057-1976/ab9dd2.

9. Epstein N. A review: reduced reoperation rate for multilevel lumbar laminectomies with noninstrumented versus instrumented fusions. Surg Neurol Int. 2016;7:337. https://doi.org/10.4103/2152-7806.182546.

10. ZdeblickTA. A Prospective, Randomized Study of Lumbar Fusion. Spine (Phila Pa 1976). 1993;18:983-91. https://doi.org/10.1097/00007632-19930 6150-00006.

11. Fritzell P, Hägg O, Wessberg P, Nordwall A. Chronic Low Back Pain and Fusion: A Comparison of Three Surgical Techniques. Spine (Phila Pa 1976). 2002;27:1131-41. https://doi.org/10.1097/00007632-200206010-00002.

12. Ozer AF, Oktenoglu T, Egemen E, Sasani M, Yilmaz A, Erbulut DU, et al. Lumbar single-level dynamic stabilization with semi-rigid and full dynamic systems: a retrospective clinical and radiological analysis of 71 patients. Clin Orthop Surg. 2017;9:310-6. https://doi.org/10.4055/cios. 2017.9.3.310.

13. Sénégas J, Vital J-M, Pointillart V, Mangione P. Clinical evaluation of a lumbar interspinous dynamic stabilization device (the Wallis system) with a 13-year mean follow-up. Neurosurg Rev. 2009;32:335-42. https://doi. org/10.1007/s10143-009-0199-z.

14. Wilke H-J, Drumm J, Häussler K, Mack C, Steudel W-I, Kettler A. Biomechanical effect of different lumbar interspinous implants on flexibility and intradiscal pressure. Eur Spine J. 2008;17:1049-56. https://doi.org/10. 1007/s00586-008-0657-2.

15. Rana M, Roy S, Biswas P, Biswas SK, Biswas JK. Design and development of a novel expanding flexible rod device (FRD) for stability in the lumbar spine: a finite-element study. Int J Artif Organs. 2020;43:803-10. https:// doi.org/10.1177/0391398820917390.

16. Jain $P$, Khan MR. Prediction of biomechanical behavior of lumbar vertebrae using a novel semi-rigid stabilization device. Proc Inst Mech Eng Part H J Eng Med. 2019;233:849-57. https://doi.org/10.1177/0954411919856497.

17. Li C, Liu L, Shi J-Y, Yan K-Z, Shen W-Z, Yang Z-R. Clinical and biomechanical researches of polyetheretherketone (PEEK) rods for semi-rigid lumbar fusion: a systematic review. Neurosurg Rev. 2018;41:375-89. https://doi. org/10.1007/s10143-016-0763-2.

18. Korovessis P, Papazisis Z, Koureas G, Lambiris E. Rigid, Semirigid Versus Dynamic Instrumentation for Degenerative Lumbar Spinal Stenosis. Spine (Phila Pa 1976). 2004;29:735-42. https://doi.org/10.1097/01.BRS. 0000112072.83196.0F.

19. Harris BM, Hilibrand AS, Savas PE, Pellegrino A, Vaccaro AR, Siegler S, et al. Transforaminal Lumbar Interbody Fusion. Spine (Phila Pa 1976). 2004;29:E65-70. https://doi.org/10.1097/01.BRS.0000113034 74567.86.

20. Bothmann M, Kast E, Boldt GJ, Oberle J. Dynesys fixation for lumbar spine degeneration. Neurosurg Rev. 2008;31:189-96. https://doi.org/10.1007/ s10143-007-0101-9.

21. Gornet MF, Chan FW, Coleman JC, Murrell B, Nockels RP, Taylor BA, et al. Biomechanical assessment of a PEEK rod system for semi-rigid fixation of lumbar fusion constructs. J Biomech Eng. 2011;133:081009. https://doi. org/10.1115/1.4004862.

22. Biswas JK, Roy S, Rana M, Halder S. A comparison of rigid, semi-rigid and flexible spinal stabilization devices: a finite element study. Proc Inst Mech Eng Part H J Eng Med. 2019;233:1292-8. https://doi.org/10.1177/09544 11919880694. 
23. Hsieh Y-Y, Tsuang F-Y, Kuo Y-J, Chen C-H, Chiang C-J, Lin C-L. Biomechanical analysis of single-level interbody fusion with different internal fixation rod materials: a finite element analysis. BMC Musculoskelet Disord. 2020;21:100. https://doi.org/10.1186/s12891-020-3111-1.

24. Winn $H$. Youmans and Winn Neurological Surgery.

25. Parchi PD, Evangelisti G, Vertuccio A, Piolanti N, Andreani L, Cervi V, et al. Biomechanics of Interspinous devices. Biomed Res Int. 2014;2014:1-7. https://doi.org/10.1155/2014/839325.

26. Phillips FM, Voronov LI, Gaitanis IN, Carandang G, Havey RM, Patwardhan AG. Biomechanics of posterior dynamic stabilizing device (DIAM) after facetectomy and discectomy. Spine J. 2006;6:714-22. https://doi.org/10. 1016/j.spinee.2006.02.003

27. Tsai K-J, Murakami H, Lowery GL, Hutton WC. A biomechanical evaluation of an interspinous device (Coflex) used to stabilize the lumbar spine. J Surg Orthop Adv. 2006;15:167-72 http://www.ncbi.nlm.nih.gov/pubmed/17087886.

28. Lafage V, Gangnet N, Sénégas J, Lavaste F, Skalli W. New Interspinous Implant Evaluation Using an In Vitro Biomechanical Study Combined With a Finite-Element Analysis. Spine (Phila Pa 1976). 2007;32:1706-13. doi:https://doi.org/10.1097/BRS.0b013e3180b9f429.

29. Gonzalez-Blohm SA, Doulgeris JJ, Aghayev K, Lee WE, Volkov A, Vrionis FD. Biomechanical analysis of an interspinous fusion device as a stand-alone and as supplemental fixation to posterior expandable interbody cages in the lumbar spine. J Neurosurg Spine. 2014;20:209-19. https://doi.org/10. 3171/2013.10.SPINE13612.

30. Kuchta J, Sobottke R, Eysel P, Simons P. Two-year results of interspinous spacer (X-stop) implantation in 175 patients with neurologic intermittent claudication due to lumbar spinal stenosis. Eur Spine J. 2009;18:823-9. https://doi.org/10.1007/s00586-009-0967-z.

31. Watanabe K, Matsumoto M, Ikegami T, Nishiwaki Y, Tsuji T, Ishii K, et al. Reduced postoperative wound pain after lumbar spinous process-splitting laminectomy for lumbar canal stenosis: a randomized controlled study. J Neurosurg Spine. 2011;14:51-8. https://doi.org/10.3171/2010.9. SPINE09933.

32. Pfirrmann CWA, Metzdorf A, Zanetti M, Hodler J, Boos N. Magnetic Resonance Classification of Lumbar Intervertebral Disc Degeneration. Spine (Phila Pa 1976). 2001;26:1873-8. https://doi.org/10.1097/00007632-20010 9010-00011.

33. Polikeit A, Ferguson SJ, Nolte LP, Orr TE. Factors influencing stresses in the lumbar spine after the insertion of intervertebral cages: finite element analysis. Eur Spine J. 2003;12:413-20. https://doi.org/10.1007/ s00586-002-0505-8.

34. Wong C-E, Hu H-T, Hsieh M-P, Huang K-Y. Optimization of three-level cervical hybrid surgery to prevent adjacent segment disease: a finite element study. Front Bioeng Biotechnol. 2020;8:154. https://doi.org/10.3389/ fbioe.2020.00154.

35. Wong C-E, Hu H-T, Tsai C-H, Li J-L, Hsieh C-C, Huang K-Y. Comparison of posterior fixation strategies for thoracolumbar burst fracture: a finite element study. J Biomech Eng. 2021. https://doi.org/10.1115/1.4050537.

36. Benneker LM, Heini PF, Anderson SE, Alini M, Ito K. Correlation of radiographic and MRI parameters to morphological and biochemical assessment of intervertebral disc degeneration. Eur Spine J. 2005;14:27-35. https://doi.org/10.1007/s00586-004-0759-4.

37. Antoniou J, Epure LM, Michalek AJ, Grant MP, latridis JC, Mwale F. Analysis of quantitative magnetic resonance imaging and biomechanical parameters on human discs with different grades of degeneration. J Magn Reson Imaging. 2013;38:1402-14. https://doi.org/10.1002/jmri.24120.

38. Shan Z, Li S, Liu J, Mamuti M, Wang C, Zhao F. Correlation between biomechanical properties of the annulus fibrosus and magnetic resonance imaging (MRI) findings. Eur Spine J. 2015;24:1909-16. https://doi.org/10. 1007/s00586-015-4061-4.

39. Polikeit A, Nolte LP, Ferguson SJ. Simulated influence of osteoporosis and disc degeneration on the load transfer in a lumbar functional spinal unit. J Biomech. 2004;37:1061-9. https://doi.org/10.1016/j.jbiomech.2003.11. 018.

40. Goel VK, Park H, Kong W. Investigation of vibration characteristics of the ligamentous lumbar spine using the finite element approach. J Biomech Eng. 1994;116:377-83. https://doi.org/10.1115/1.2895787.

41. Pearcy M, Portek I, Shepherd J. Three-dimensional X-ray analysis of normal movement in the lumbar spine. Spine (Phila Pa 1976). 1984;9:294-7. https://doi.org/10.1097/00007632-198404000-00013.
42. Pearcy MJ, Tibrewal SB. Axial rotation and lateral bending in the normal lumbar spine measured by three-dimensional radiography. Spine (Phila Pa 1976). 1984;9:582-7. doi:https://doi.org/10.1097/00007632-19840 9000-00008.

43. White AA, Panjabi MM. The basic kinematics of the human spine. A review of past and current knowledge. Spine (Phila Pa 1976). 1978;3:1220. doi:https://doi.org/10.1097/00007632-197803000-00003.

44. Yamamoto I, Panjabi MM, Crisco T, Oxland T. Three-dimensional movements of the whole lumbar spine and lumbosacral joint. Spine (Phila Pa 1976). 1989;14:1256-60. doi:https://doi.org/10.1097/00007632-19891 1000-00020.

45. Cunningham BW, Kotani Y, McNulty PS, Cappuccino A, McAfee PC. The effect of spinal destabilization and instrumentation on lumbar intradiscal pressure: an in vitro biomechanical analysis. Spine (Phila Pa 1976). 1997;22:2655-63. doi:https://doi.org/10.1097/00007632-19971 $1150-00014$.

46. Brinckmann P, Grootenboer $\mathrm{H}$. Change of disc height, radial disc bulge, and intradiscal pressure from discectomy. An in vitro investigation on human lumbar discs. Spine (Phila Pa 1976). 1991;16:641-6. doi:https://doi. org/10.1097/00007632-199106000-00008.

47. Wilke HJ, Wolf S, Claes LE, Arand M, Wiesend A. Influence of varying muscle forces on lumbar intradiscal pressure: an in vitro study. J Biomech. 1996;29:549-55. https://doi.org/10.1016/0021-9290(95)00037-2.

48. Li C, He Q, Tang Y, Ruan D. The fate of adjacent segments with preexisting degeneration after lumbar posterolateral fusion: the influence of degenerative grading. Eur Spine J. 2015;24:2468-73. https://doi.org/10. 1007/s00586-015-3921-2.

49. Saleem S, Aslam HM, Rehmani MAK, Raees A, Alvi AA, Ashraf J. Lumbar disc degenerative disease: disc degeneration symptoms and magnetic resonance image findings. Asian Spine J. 2013;7:322-34. https://doi.org/ 10.4184/asj.2013.7.4.322.

50. Ravindra VM, Senglaub SS, Rattani A, Dewan MC, Härtl R, Bisson E, et al. Degenerative lumbar spine disease: estimating global incidence and worldwide volume. Glob spine J. 2018;8:784-94. https://doi.org/10.1177/ 2192568218770769.

51. Chun DS, Baker KC, Hsu WK. Lumbar pseudarthrosis: a review of current diagnosis and treatment. Neurosurg Focus. 2015;39:E10. https://doi.org/ 10.3171/2015.7.FOCUS15292

52. Reid JJ, Johnson JS, Wang JC. Challenges to bone formation in spinal fusion. J Biomech. 2011;44:213-20. https://doi.org/10.1016/j.jbiomech. 2010.10.021.

53. Narimani M, Arjmand N. Three-dimensional primary and coupled range of motions and movement coordination of the pelvis, lumbar and thoracic spine in standing posture using inertial tracking device. J Biomech. 2018;69:169-74. https://doi.org/10.1016/j.jbiomech.2018.01.017.

54. Wang H, Ma L, Yang D, Wang T, Liu S, Yang S, et al. Incidence and risk factors of adjacent segment disease following posterior decompression and instrumented fusion for degenerative lumbar disorders. Medicine (Baltimore). 2017;96:e6032. doi:https://doi.org/10.1097/MD.0000000000 006032.

55. Pan A, Hai Y, Yang J, Zhou L, Chen X, Guo H. Adjacent segment degeneration after lumbar spinal fusion compared with motion-preservation procedures: a meta-analysis. Eur Spine J. 2016;25:1522-32. https://doi. org/10.1007/s00586-016-4415-6.

56. Doblaré M, García JM, Gómez MJ. Modelling bone tissue fracture and healing: a review. Eng Fract Mech. 2004;71:1809-40.

57. Kelsey JL. An epidemiological study of acute herniated lumbar intervertebral discs. Rheumatol Rehabil. 1975;14:144-59. https://doi.org/10.1093/ rheumatology/14.3.144.

58. Pattin CA, Caler WE, Carter DR. Cyclic mechanical property degradation during fatigue loading of cortical bone. J Biomech. 1996;29:69-79. https://doi.org/10.1016/0021-9290(94)00156-1.

59. Kothe R, Rüther W, Schneider E, Linke B. Biomechanical analysis of transpedicular screw fixation in the subaxial cervical spine. Spine (Phila Pa 1976). 2004;29:1869-75. doi:https://doi.org/10.1097/01.brs.0000137287.67388.0b.

\section{Publisher's Note}

Springer Nature remains neutral with regard to jurisdictional claims in published maps and institutional affiliations. 